\title{
Tuberculin skin test and interferon-gamma release assay values are associated with antimicrobial peptides expression in polymorphonuclear cells during latent tuberculous infection
}

\author{
Julio E Castañeda-Delgado, ${ }^{1,}$, Alberto Cervantes-Villagrana ${ }^{1,3}$, Carmen J Serrano-Escobedo', \\ Isabel Frausto-Lujan', Cesar Rivas-Santiago ${ }^{4}$, Jose A Enciso-Moreno', Bruno Rivas-Santiago ${ }^{1 /+}$ \\ ${ }^{1}$ Medical Research Unit of Zacatecas, Mexican Institute of Social Security, Zacatecas, Mexico ${ }^{2}$ Department of Immunology, \\ Faculty of Medicine, Autonomous University of San Luis Potosí, San Luis Potosi, Mexico ${ }^{3}$ Academic Unit of Chemical Sciences, \\ Autonomous University of Zacatecas, Zacatecas, México ${ }^{4}$ Department of Environmental and Occupational Health, Center for Global \\ Public Health, School of Public Health, University of Medicine and Dentistry New Jersey, Piscataway, NJ, USA
}

It has been reported that patients with progressive tuberculosis (TB) express abundant amounts of the antimicrobial peptides (AMPS) cathelicidin (LL-37) and human neutrophil peptide-1 (HNP-1) in circulating cells, whereas latent TB infected donors showed no differences when compared with purified protein derivative (PPD) and QuantiFERON ${ }^{\circledR}-T B$ Gold (QFT)-healthy individuals. The aim of this study was to determine whether LL-37 and HNP-1 production correlates with higher tuberculin skin test (TST) and QFT values in TB household contacts. Twenty-six TB household contact individuals between 26-58 years old TST and QFT positive with at last two years of latent TB infection were recruited. AMPs production by polymorphonuclear cells was determined by flow cytometry and correlation between TST and QFT values was analysed. Our results showed that there is a positive correlation between levels of HNP-1 and LL-37 production with reactivity to TST and/or QFT levels. This preliminary study suggests the potential use of the expression levels of these peptides as biomarkers for progression in latent infected individuals.

Key words: tuberculosis - latent infection - antimicrobial peptides - tuberculin skin test - QuantiFERON - biomarker

\begin{abstract}
Mycobacterium tuberculosis (Mtb) is a pathogen that may cause either progressive disease or latent infection in humans. Household contacts of pulmonary tuberculosis (TB) patients are at high risk of Mtb infection and early disease development (Fox et al. 2013). Intriguingly most of the household patients develop latent TB instead, which is clinically asymptomatic, however a percentage of them develop progressive disease afterwards. Albeit the risk to develop active TB is higher in household contacts, does not exist any biomarker for progression. The use of the tuberculin skin test (TST) and interferon-gamma (IFN- $\gamma$ ) release assays to diagnose latent TB infection has been established and validated in large studies, which observed that persons with a positive values for these tests had a higher risk of developing active TB compared with negative-value persons (Fox et al. 2013). The QuantiFERON ${ }_{-}{ }_{-}$ TB Gold (QFT) is one such IFN- $\gamma$ release assay commercially available, which has been adopted by many countries as an alternative to TST or as part of a two-step approach which uses both tests. QFT-In Tube (QFT-IT) uses early secreted antigenic target 6 (ESAT-6), culture filtrate protein 10 (CFP-10) and antigen 7.7 of Mtb as stimuli to determine if $\mathrm{T}$ cells in whole blood are sensitised to
\end{abstract}

doi: 10.1590/0074-0276140348

Financial support: IMSS (FIS/IMSS/PROT/G11/955), CONACYT

(CONACYT-SALUD-2008-C01-87657)

+ Corresponding author: rondo_vm@hotmail.com

Received 1 July 2013

Accepted 18 November 2013 such antigens thus indicating prior exposure and/or evidence of latent TB infection (Bakir et al. 2008).

Antimicrobial peptides (AMPs) represent an important part of the innate immunity, furthermore they are considered as a link with adaptive immune response in several infectious diseases such as TB (Hancock et al. 2012, Rivas-Santiago et al. 2013). Polymorphonuclear leukocytes (PMN) bear a huge amount of AMPs mainly human neutrophil peptide-1 (HNP-1) and cathelicidin (LL-37) which are released both for direct antimicrobial activity and for immunomodulatory functions (Cederlund et al. 2011). During their journey from the blood stream to sites of inflammation PMN release wide panoply of granule proteins including AMPs which altogether allow direct activation and subsequent migration of monocytes. In addition, AMPs granule proteins exert direct chemotactic effects, a mechanism which is of special importance in the early recruitment of inflammatory monocytes and initial activation of antigen specific $\mathrm{CD}^{+} \mathrm{T}$ cells (Soehnlein et al. 2009, Blomgran \& Ernst 2011).

Previous studies by our group have shown that patients with progressive TB express abundant amounts of LL-37 and HNP-1, whereas latent TB infected donors showed no differences when compared with purified protein derivative (PPD) and QFT-healthy individuals (Gonzalez-Curiel et al. 2011). Since it has been reported that QFT and TST conversion is indicative of an approximately eight fold higher risk of progression to TB disease (compared with non-converters) in a highTB burden population (Diel et al. 2012, Machingaidze et al. 2012, Trajman et al. 2013); we sought to determine whether LL-37 and HNP-1 production correlates with higher TST and QFT values in TB household contacts. 


\section{SUBJECTS, MATERIALS AND METHODS}

Subjects - This study was approved by the Ethical and Scientific Committees of the Mexican Institute of Social Security (R-2008-3301-15). Twenty-six TB household contact individuals between 26-58 years old were recruited between March-June 2012. TB household contacts included individuals with no TB diagnosis confirmed who lived in the same household as the index case or visited the house at least once per week, adding a total cumulative indoor exposure with shared air of at least $40 \mathrm{~h}$ within the three months prior to the index case diagnosis to maximise the identification of possibly infected contacts. Contacts were first visited and interviewed between March-December 2010 (1-37 months after the index case was diagnosed) using a standardised questionnaire. Thus TST and QFT positive individuals on the present study had at least two years of presumable latent TB infection. To discard active TB infection, contacts were submitted to clinical examination, including sputum culture. None of the patients had any systematic or autoimmune disease, neither steroids nor other hormone therapy. All patients were human immunodeficiency virus negative. Written informed consent was obtained from each volunteer prior to blood sample drawing.

TST and QFT-IT - All blood samples for QFT-IT assays and flow cytometry analysis were drawn before the application of the skin test. Each TST was performed by intradermal injection of five tuberculin units of PPD TUBERSOL (Aventis Pasteur Ltd, Canada) into the forearm using the Mantoux technique. Reactions were evaluated $48 \mathrm{~h}$ after injection, with a positive result defined as an induration of $\geq 10 \mathrm{~mm}$ in the transverse diameter read by medical trained personnel. The ELISA-based QFT-IT assay (Cellestis Inc, Valencia, CA, USA) was performed in two stages, according to the manufacturer's instructions. Briefly, 1-mL aliquots of blood were drawn directly into three blood collection tubes, one containing (negative control), one containing $\mathrm{T}$ cell mitogen (positive control) and one containing Mtb-specific antigens, including ESAT-6, CFP-10 and TB7.7 (TB-antigen tube). Following overnight incubation, $200 \mu \mathrm{L}$ of plasma were removed from each tube and the concentration of IFN- $\gamma$ was determined by ELISA (Cellestis Inc), with a positive response defined as a TB antigen-nil IFN- $\gamma$ concentration $\geq 0.35 \mathrm{IU} / \mathrm{mL}$. The individuals were categorised into two groups according to their reactivity to QFT, meaning as positive or negative to the test.

Cell purification - Blood samples were processed in a time frame no longer than $2 \mathrm{~h}$ after blood drawing. Polymorphonuclear cells were isolated from peripheral blood by using Ficoll-Paque PLUS (GE Healthcare, Uppsala, Sweden) and lysis buffer with ammonium chloride. Once isolated, cells were washed twice with sterile phosphate buffer saline and their number and viability was determined by direct counting and by trypan blue exclusion assay. Purity of polymorphonuclear cells was determined by flow cytometry (>90\%).
Analysis of AMPs by flow cytometry - AMPs production by polymorphonuclear cells was determined as follows: $2 \times 10^{5}$ cells were treated with fixation and permeabilisation solution (BD Pharmigen, San Jose, CA, USA). The cells were then washed with perm/wash buffer (BD Pharmigen) and incubated with a goat antiLL-37 polyclonal antibody (Santa Cruz Biotechnology, Santa Cruz, CA, USA) or goat polyclonal anti-HNP-1 antibody (Serotec, Oxford, UK). The detection of these antibodies was made with a rabbit polyclonal anti-goat labelled with phycoerithrin (Santa Cruz Biotechnology). After washing, the cells were analysed in a FACS CANTO II flow cytometer (Becton \& Dickinson, NJ, USA) with the FACS DIVA software (Becton Dickinson).

Statistical analysis - Data from the clinical features of the participants, the proportion of positive cells as well as the mean fluorescence intensities were analysed through a Kolmogorov-Smirnov normality test. After this, one of the following tests was performed to determine if significant differences existed among groups: Mann-Whitney $U$ test, Student's $t$ test with and without Welch correction, which was indicated in each figure accordingly. Also, a non-parametric Spearman correlation was performed in order to determine the possible association between QFT-IT and TST continuous values and the production of HNP-1 and LL-37. $p$ values of $<0.05$ were considered statistically significant.

\section{RESULTS}

Clinical analysis - We obtained samples from 26 individuals, which according to the TST and QFT results, were divided into two groups, those who were positive to TST and QFT ( $\mathrm{n}=12$, further referred as $\left.\mathrm{QFT}^{+}\right)$and those who were negative for both tests $(n=14$, further referred as QFT). The clinical features of the participants are summarised in Table. None of the clinical features show statistical differences, except for their reactivity to TST and QFT $(p<0.001)$. All individuals were negative for Mtb culture.

$A M P s$ are increased in QFT positive individuals Polymorphonuclear cells were purified and analysed by flow cytometry to determine the proportion and production of the AMPs HNP-1 and LL-37 in the two different groups. Results showed that there is an increased proportion of LL-37 positive cells in the $\mathrm{QFT}^{+}$individuals $(\mathrm{p}=$ 0.0184) when compared with QFT individuals (Fig. 1A), which correlates with the mean fluorescence intensity (MFI), suggesting an increased production of peptide on a per-cell basis (Fig. 1B) $(\mathrm{p}=0.0116)$. HNP-1 positive cells analysis showed no differences between groups ( $\mathrm{p}$ $=0.3413)($ Fig. 1C). However, significant differences ( $p$ $=0.0043$ ) were observed when analysed for the MFI between groups as shown in Fig. 1D.

AMPs production correlates with QFT and TST reactivity values - To determine whether positive values of QFT and TST correlated with increased production of HNP-1 and LL-37 both variables were analysed for each individual. As shown in Fig. 2A, LL-37 production is correlated with TST reactivity, as well there is an association 
TABLE

Clinical features of participants

\begin{tabular}{|c|c|c|c|}
\hline & $\begin{array}{l}\text { QFT and TST negative } \\
\qquad(\mathrm{n}=14) \\
(\text { mean } \pm \mathrm{SD})\end{array}$ & $\begin{array}{l}\text { QFT and TST positive } \\
\qquad(\mathrm{n}=12) \\
(\text { mean } \pm \mathrm{SD})\end{array}$ & $\mathrm{p}$ \\
\hline TST (mm) & $5.93 \pm 7.46$ & $20.25 \pm 3.96$ & $<0.001^{a}$ \\
\hline QFT (UI/mL) & $0.03 \pm 0.06$ & $6.81 \pm 3.42$ & $<0.001^{b}$ \\
\hline Age (years) & $49.00 \pm 23.49$ & $41.92 \pm 15.25$ & $0.3801^{c}$ \\
\hline Weight (Kg) & $73.31 \pm 18.47$ & $68.18 \pm 10.88$ & $0.4076^{c}$ \\
\hline Height (m) & $1.62 \pm 0.12$ & $1.60 \pm .06$ & $0.5749^{a}$ \\
\hline Hip (cm) & $107.36 \pm 10.92$ & $102.17 \pm 7.72$ & $0.1816^{c}$ \\
\hline Waist (cm) & $92.14 \pm 13.56$ & $88.25 \pm 11.96$ & $0.4488^{c}$ \\
\hline Body mass index $\left(\mathrm{kg} / \mathrm{m}^{2}\right)$ & $27.50 \pm 4.82$ & $26.49 \pm 3.89$ & $0.5642^{c}$ \\
\hline Waist to hip ratio & $0.86 \pm 0.08$ & $0.86 \pm .07$ & $0.8948^{c}$ \\
\hline Sex (male/female) & $4 / 10$ & $4 / 8$ & Not available \\
\hline Haemoglobin $(\mathrm{g} / \mathrm{dL})$ & $14.27 \pm 1.36$ & $14.49 \pm 1.71$ & $0.7179^{c}$ \\
\hline Leucocytes $\left(1 \times 10^{3}\right.$ cell $\left./ \mu \mathrm{L}\right)$ & $6.39 \pm 1.80$ & $6.82 \pm 1.82$ & $0.6996^{b}$ \\
\hline Lymphocytes $\left(1 \times 10^{3}\right.$ cell $\left./ \mu \mathrm{L}\right)$ & $2.42 \pm 0.95$ & $2.29 \pm .45$ & $0.8201^{b}$ \\
\hline Glucose $(\mathrm{mg} / \mathrm{dL})$ & $78.64 \pm 27.65$ & $96.48 \pm 75.62$ & $0.9180^{b}$ \\
\hline Creatinine (mg/dL) & $0.81 \pm 0.27$ & $0.75 \pm 0.17$ & $0.8360^{b}$ \\
\hline Cholesterol (mg/dL) & $196.29 \pm 35.05$ & $196.49 \pm 31.88$ & $0.9877^{c}$ \\
\hline Triglycerides (mg/dL) & $164.31 \pm 79.36$ & $235.88 \pm 119.14$ & $0.1308^{b}$ \\
\hline
\end{tabular}

$a$ : Welch corrected unpaired t test; $b$ : Mann-Whitney $U$ test; $c$ : unpaired $t$ test; QFT: QuantiFERON ${ }^{\circledR}-$ TB Gold; SD: standard deviation; TST: tuberculin skin test.
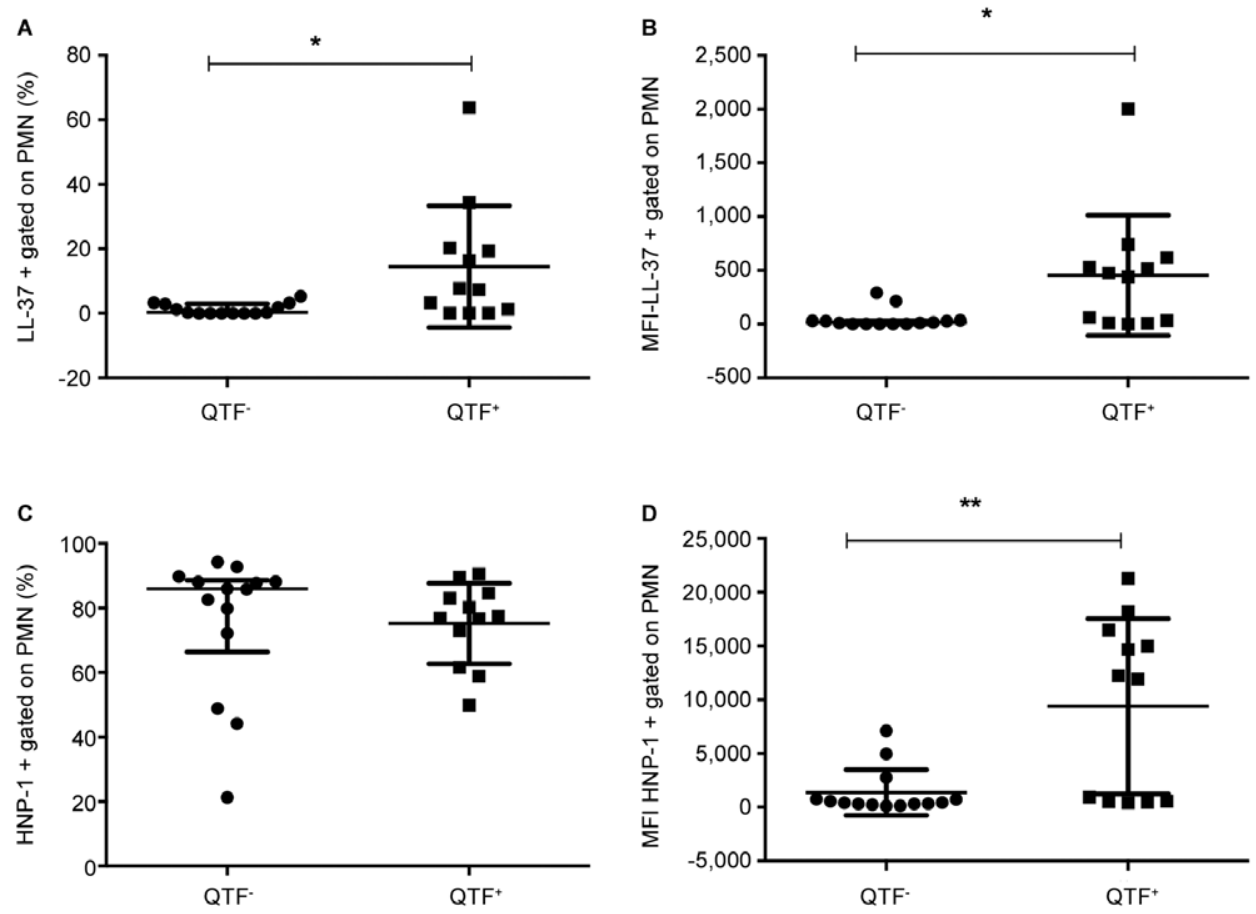

Fig. 1: cathelicidin (LL-37) and human neutrophil peptide-1 (HNP-1) production in polymorphonuclear cells is increased in tuberculosis latently infected individuals. The percentage of HNP-1 ${ }^{+}$and LL- $37^{+}$cells was determined by analysis in a flow cytometer in polymorphonuclear leukocytes (PMN) gated cells (A, C). Also, LL37 ${ }^{+}(\mathrm{B})$ and HNP-1 ${ }^{+}$(D) cells mean fluorescence intensity (MFI) was analysed. Data is presented as median \pm interquartile range. Group comparisons were made by means of a non-parametric Mann Whitney $U$ test. In QuantiFERON ${ }^{\circledR}-\mathrm{TB}$ Gold $(\mathrm{QFT})^{-}, \mathrm{n}=14$ and in $\mathrm{QFT}^{+}, \mathrm{n}=12$. $\mathrm{p}=0.05$ or lower were considered statistically significant. 
A

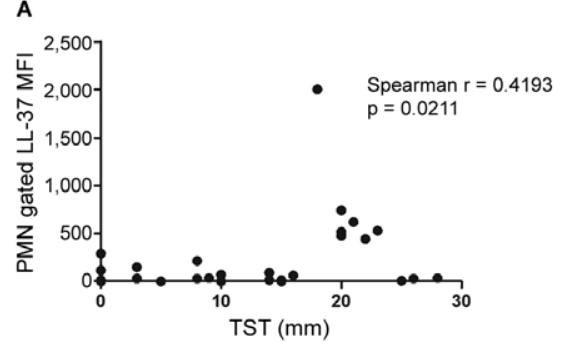

C

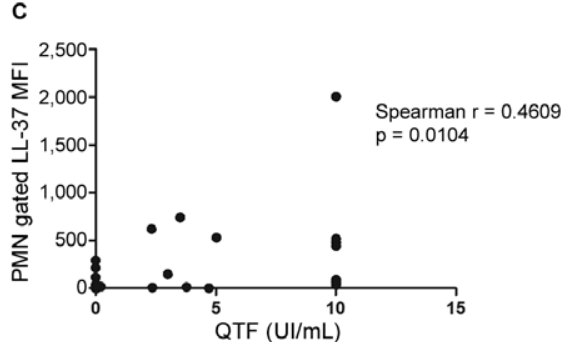

B

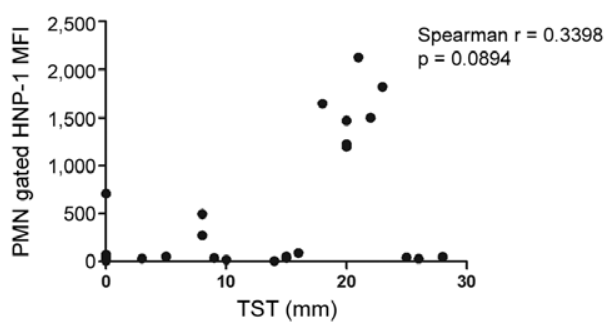

D

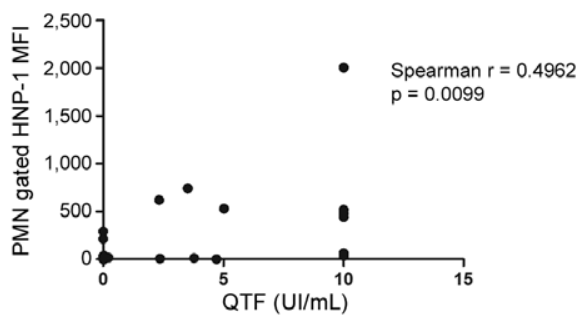

Fig. 2: cathelicidin (LL-37) and human neutrophil peptide-1 (HNP-1) correlated with QuantiFERON ${ }^{\circledR}-\mathrm{TB}$ Gold (QFT) and tuberculin skin test (TST) continuous values. Correlation between TST positivity with LL-37 (A) and HNP-1 (B) mean fluorescence intensity (MFI) was analysed. Also, QFT positivity was correlated with LL-37 (C) and HNP-1 (D) MFI. The Spearman correlation coefficient and the level of significance are shown for each graph. $\mathrm{p}$ values of more than 0.05 were considered not statistically significant, $\mathrm{n}=26$.

with HNP-1 production (Fig. 2B). Similarly, when correlated with QFT positivity a positive correlation was found for both LL-37 and HNP-1 (Fig. 2C, D, respectively).

\section{DISCUSSION}

Previous studies by our group have demonstrated the importance of AMPs to contain TB infection either during primary or latent infection (Rivas-Santiago et al. 2006, 2008a). Within this group of AMPs it has been reported that $\beta$-defensins have direct antimicrobial activity against Mtb, however other AMPs such as LL-37 and HNP-1, mainly produced by neutrophils are included in the Mtb containing process through a wide variety of immunomodulatory functions (Martineau et al. 2007, Rivas-Santiago et al. 2008b, Castañeda-Delgado et al. 2010). During the infection, neutrophils accumulate at sites of mycobacterial challenge and rapidly phagocytose bacilli and release several neutrophil-derived chemokines, cytokines, granule products, HNP-1 and LL-37 that can drive monocyte recruitment from blood. After monocyte recruitment and maturation, cytokine secretion by both neutrophils and macrophages might drive further accumulation and activation of both cell types leading to inflammation (Martineau et al. 2007, Soehnlein et al. 2009, Lowe et al. 2012). A previous study reported that LL-37 and HNP-1 gene expression was highly increased above 20 -fold in patients with active TB compared with healthy volunteers and latent TB infected individuals, these data indicate that these peptides might be increasing during progression of disease (Gonzalez-Curiel et al. 2011). Other studies have linked the presence of high amounts of neutrophils in granuloma with increasing pneumonia and low mycobacterial control which lead to an active disease (Eum et al. 2010), thus we hypothesise that since AMPs producing cells are increased while TB become active, should be a positive correlation with HNP-1 and LL-37. Recent studies suggest that the use of QFT conversion is indicative of an approximately eight-fold higher risk of progression to TB disease (compared with non-converters) within two years of conversion in a cohort of adolescents in a highTB burden population (Machingaidze et al. 2012) and in a low incidence area (Bergot et al. 2012), we sought to determine if there was a correlation between the expression of HNP-1 and LL-37 with TST and QFT continuous values. Our results indicated that individuals who have positive values to TST and QFT had statistical significances regarding production of LL-37 and HNP-1, in the same line we found a positive correlation between expression of AMPs and the continuous values for QFT and TST. Altogether these data suggest that during gradual reactivation process there is an influx of PMNs into the lung to contain bacilli growth and to recruit monocytes. One of the main activators markers in neutrophils might be LL-37, since has been reported that increment of LL37 in PMN promotes the generation of reactive oxygen species dose and time-dependently, via nicotinamide adenine dinucleotide phosphate oxidase activation and intracellular $\mathrm{Ca}\left(2^{+}\right)$mobilisation. Besides LL-37 induces both mRNA expression and protein release of alphadefensins neutrophil peptide 1-3 (Zheng et al. 2007) and promotes monocyte adhesion (Wantha et al. 2013) during infection. Furthermore, LL-37 is a keystone for effective antimicrobial activity since it controls the release of inflammatory mediators while increasing antimicrobial activity of neutrophils (Alalwani et al. 2010). 
The main limitation of this study is that it is a crosssectional study and the individuals with latent TB have not been followed longitudinally to determine whether they develop disease. The reduced sample size is another handicap of the study; however it is good enough to observe tendencies and to plan further studies with a bigger population. Finally, the possibility that other infections or autoimmune diseases could interfere with the levels of these peptides needs to be elucidated.

All together our results indicate that AMPs levels positive correlates with TST and QFT values. Due there is not an accurate biomarker of progression for TB in latent infected individuals, this preliminary study suggests the use of HNPs and LL-37 as promising biomarkers for progression, however further studies need to be done to strength this results.

\section{REFERENCES}

Alalwani SM, Sierigk J, Herr C, Pinkenburg O, Gallo R, Vogelmeier C, Bals R 2010. The antimicrobial peptide LL-37 modulates the inflammatory and host defense response of human neutrophils. Eur J Immunol 40: 1118-1126.

Bakir M, Millington KA, Soysal A, Deeks JJ, Efee S, Aslan Y, Dosanjh DP, Lalvani A 2008. Prognostic value of a T-cell-based, interferon-gamma biomarker in children with tuberculosis contact. Ann Intern Med 149: 777-787.

Bergot E, Haustraete E, Malbruny B, Magnier R, Salaun MA, Zalcman G 2012. Observational study of QuantiFERON(R)-TB Gold In-Tube assay in tuberculosis contacts in a low incidence area. PLOS ONE 7: e43520.

Blomgran R, Ernst JD 2011. Lung neutrophils facilitate activation of naïve antigen-specific $\mathrm{CD} 4^{+} \mathrm{T}$ cells during Mycobacterium tuberculosis infection. J Immunol 186: 7110-7119.

Castañeda-Delgado J, Hernández-Pando R, Serrano CJ, Aguilar-León D, León-Contreras J, Rivas-Santiago C, Méndez R, GonzálezCuriel I, Enciso-Moreno A, Rivas-Santiago B 2010. Kinetics and cellular sources of cathelicidin during the course of experimental latent tuberculous infection and progressive pulmonary tuberculosis. Clin Exp Immunol 161: 542-550.

Cederlund A, Gudmundsson GH, Agerberth B 2011. Antimicrobial peptides important in innate immunity. FEBS J 278: 3942-3951.

Diel R, Loddenkemper R, Nienhaus A 2012. Predictive value of interferon-gamma release assays and tuberculin skin testing for progression from latent TB infection to disease state: a metaanalysis. Chest 142: 63-75.

Eum SY, Kong JH, Hong MS, Lee YJ, Kim JH, Hwang SH, Cho SN, Via LE, Barry CE 3rd 2010. Neutrophils are the predominant infected phagocytic cells in the airways of patients with active pulmonary TB. Chest 137: 122-128.
Fox GJ, Barry SE, Britton WJ, Marks GB 2013. Contact investigation for tuberculosis: a systematic review and meta-analysis. Eur Respir J 41: 140-156.

Gonzalez-Curiel I, Castañeda-Delgado J, Lopez-Lopez N, Araujo Z, Hernandez-Pando R, Gandara-Jasso B, Macias-Segura N, Enciso-Moreno A, Rivas-Santiago B 2011. Differential expression of antimicrobial peptides in active and latent tuberculosis and its relationship with diabetes mellitus. Hum Immunol 72: 656-662.

Hancock RE, Nijnik A, Philpott DJ 2012. Modulating immunity as a therapy for bacterial infections. Nat Rev Microbiol 10: 243-254.

Lowe DM, Redford PS, Wilkinson RJ, O'Garra A, Martineau AR 2012. Neutrophils in tuberculosis: friend or foe? Trends Immunol 33: $14-25$.

Machingaidze S, Verver S, Mulenga H, Abrahams DA, Hatherill M, Hanekom W, Hussey GD, Mahomed H 2012. Predictive value of recent QuantiFERON conversion for tuberculosis disease in adolescents. Am J Respir Crit Care Med 186: 1051-1056.

Martineau AR, Newton SM, Wilkinson KA, Kampmann B, Hall BM, Nawroly N, Packe GE, Davidson RN, Griffiths CJ, Wilkinson RJ 2007. Neutrophil-mediated innate immune resistance to mycobacteria. J Clin Invest 117: 1988-1994.

Rivas-Santiago B, Contreras JC, Sada E, Hernandez-Pando R 2008a. The potential role of lung epithelial cells and beta-defensins in experimental latent tuberculosis. Scand J Immunol 67: 448-452.

Rivas-Santiago B, Hernandez-Pando R, Carranza C, Juarez E, Contreras JL, Aguilar-Leon D, Torres M, Sada E 2008b. Expression of cathelicidin LL-37 during Mycobacterium tuberculosis infection in human alveolar macrophages, monocytes, neutrophils and epithelial cells. Infect Immun 76: 935-941.

Rivas-Santiago B, Sada E, Tsutsumi V, Aguilar-Leon D, Contreras JL, Hernandez-Pando R 2006. Beta-defensin gene expression during the course of experimental tuberculosis infection. $J$ Infect Dis 194: 697-701.

Rivas-Santiago CE, Hernandez-Pando R, Rivas-Santiago B 2013. Immunotherapy for pulmonary TB: antimicrobial peptides and their inducers. Immunotherapy 5: 1117-1126.

Soehnlein O, Weber C, Lindbom L 2009. Neutrophil granule proteins tune monocytic cell function. Trends Immunol 30: 538-546.

Trajman A, Steffen RE, Menzies D 2013. Interferon-gamma release assays versus tuberculin skin testing for the diagnosis of latent tuberculosis infection: an overview of the evidence. Pulm Med 2013: 601737.

Wantha S, Alard JE, Megens RT, van der Does AM, Doring Y, Drechsler M, Pham CT, Wang MW, Wang JM, Gallo RL, von Hundelshausen P, Lindbom L, Hackeng T, Weber C, Soehnlein O 2013. Neutrophil-derived cathelicidin promotes adhesion of classical monocytes. Circ Res 112: 792-801.

Zheng Y, Niyonsaba F, Ushio H, Nagaoka I, Ikeda S, Okumura K, Ogawa H 2007. Cathelicidin LL-37 induces the generation of reactive oxygen species and release of human alpha-defensins from neutrophils. Br J Dermatol 157: 1124-1131. 